\title{
Geometrical and band-structure effects on phonon-limited hole mobility in rectangular cross- sectional germanium nanowires
}

\section{$\operatorname{AUTHOR}(\mathrm{S})$ :}

Tanaka, H.; Mori, S.; Morioka, N.; Suda, J.; Kimoto, $T$.

\section{CITATION:}

Tanaka, H. ... [et al]. Geometrical and band-structure effects on phonon-limited hole mobility in rectangular cross-sectional germanium nanowires. Journal of Applied Physics 2014, 116(23): 235701.

ISSUE DATE:

2014-12-21

URL:

http://hdl.handle.net/2433/193264

\section{RIGHT:}

Copyright 2014 American Institute of Physics. This article may be downloaded for personal use only. Any other use requires prior permission of the author and the American Institute of Physics 


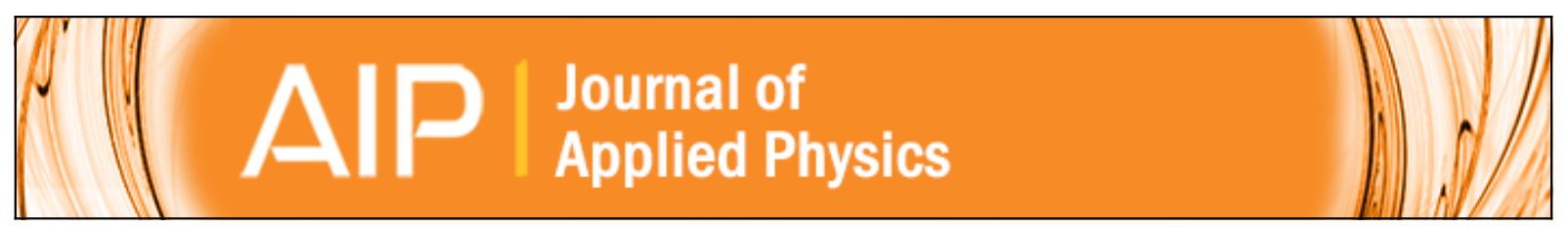

\section{Geometrical and band-structure effects on phonon-limited hole mobility in rectangular cross-sectional germanium nanowires}

H. Tanaka, S. Mori, N. Morioka, J. Suda, and T. Kimoto

Citation: Journal of Applied Physics 116, 235701 (2014); doi: 10.1063/1.4904844

View online: http://dx.doi.org/10.1063/1.4904844

View Table of Contents: http://scitation.aip.org/content/aip/journal/jap/116/23?ver=pdfcov

Published by the AIP Publishing

\section{Articles you may be interested in}

Phonon-limited low-field mobility in silicon: Quantum transport vs. linearized Boltzmann Transport Equation

J. Appl. Phys. 114, 223708 (2013); 10.1063/1.4845515

Does the low hole transport mass in 110 and 111 Si nanowires lead to mobility enhancements at high field and stress: A self-consistent tight-binding study

J. Appl. Phys. 111, 123718 (2012); 10.1063/1.4729806

Atomistic modeling of electron-phonon interaction and electron mobility in Si nanowires

J. Appl. Phys. 111, 063720 (2012); 10.1063/1.3695999

Corner effects on phonon-limited mobility in rectangular silicon nanowire metal-oxide-semiconductor field-effect transistors based on spatially resolved mobility analysis

J. Appl. Phys. 109, 113712 (2011); 10.1063/1.3592252

Quantum-confinement effect on holes in silicon nanowires: Relationship between wave function and band structure

J. Appl. Phys. 109, 064318 (2011); 10.1063/1.3552593

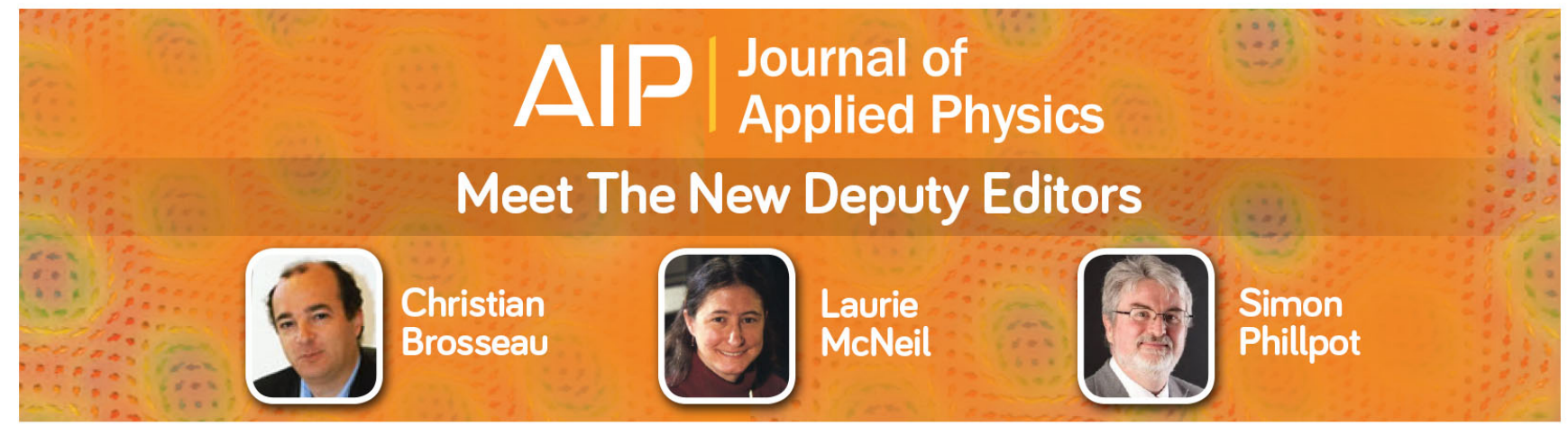




\title{
Geometrical and band-structure effects on phonon-limited hole mobility in rectangular cross-sectional germanium nanowires
}

\author{
H. Tanaka, ${ }^{\text {a) }}$ S. Mori, N. Morioka, J. Suda, and T. Kimoto \\ Department of Electronic Science and Engineering, Kyoto University, Kyoto 615-8510, Japan
}

(Received 28 October 2014; accepted 30 November 2014; published online 19 December 2014)

\begin{abstract}
We calculated the phonon-limited hole mobility in rectangular cross-sectional [001], [110], [111], and [112]-oriented germanium nanowires, and the hole transport characteristics were investigated. A tight-binding approximation was used for holes, and phonons were described by a valence force field model. Then, scattering probability of holes by phonons was calculated taking account of hole-phonon interaction atomistically, and the linearized Boltzmann's transport equation was solved to calculate the hole mobility at low longitudinal field. The dependence of the hole mobility on nanowire geometry was analyzed in terms of the valence band structure of germanium nanowires, and it was found that the dependence was qualitatively reproduced by considering an average effective mass and the density of states of holes. The calculation revealed that [110] germanium nanowires with large height along the [001] direction show high hole mobility. Germanium nanowires with this geometry are also expected to exhibit high electron mobility in our previous work, and thus they are promising for complementary metal-oxide-semiconductor (CMOS) applications. @ 2014 AIP Publishing LLC. [http://dx.doi.org/10.1063/1.4904844]
\end{abstract}

\section{INTRODUCTION}

Germanium nanowires (Ge NWs) are a promising candidate as a channel material for field-effect transistors (FETs), ${ }^{1}$ because of the high carrier mobility of $\mathrm{Ge}^{2-4}$ and the high electrostatic controllability of NW structure. ${ }^{5,6}$ Though there already exist a lot of studies on the carrier transport in $\mathrm{Ge}$ $\mathrm{NWs}^{7-9}$ including atomistic calculation of carrier mobility, ${ }^{10,11}$ the transport characteristics of Ge NWs are not sufficiently understood yet. In particular, the hole transport properties of rectangular cross-sectional Ge NWs have not been studied, to our knowledge.

In NWs, anisotropic quantum confinement effects on holes make hole states strongly dependent on the cross-sectional shape of NWs. ${ }^{12-14}$ Because rectangular cross-sectional NWs can highly utilize this anisotropy, designing the subband structure through cross-sectional shape of rectangular NWs is a potential way to achieve a high carrier transport property.

In this paper, we atomistically computed the valence band structure and phonon-limited hole mobility in rectangular cross-sectional Ge NWs with various transport orientations and cross-sectional shapes. Then, the geometry dependence of hole mobility was analyzed based on the valence band structure of Ge NWs, focusing on an averaged effective mass and the density of states of holes. Since the geometry dependence of long-channel mobility is correlated to the backscattering coefficient in quasi-ballistic transport, ${ }^{15}$ the effects of geometry and band structure on hole mobility discussed in this paper will be important even for the performance estimation of short-channel devices.

This paper is organized as follows. In Sec. II, the details of calculation methods and the structures of NWs targeted in this study are explained. In Sec. III A, the valence band

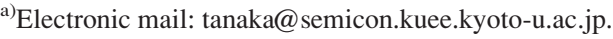

structures of several NWs are shown, and the geometry dependence of the average hole effective mass is presented. Properties of phonons in NWs are investigated in Sec. III B. Section III C shows the calculated hole mobility and discusses its geometry dependencies based on the valence band structure. Furthermore, hole density dependence of mobility is also investigated in Sec. III D. In Sec. IV, we present favorable wire geometry for hole transport and conclude this paper.

\section{CALCULATION DETAILS}

\section{A. Calculation methods}

To calculate the valence band structure and the hole states of Ge NWs, we employed a nearest-neighbor $s p^{3} d^{5} s^{*}$ tight-binding (TB) method ${ }^{16,17}$ and the TB parameters fitted to the band structure and deformation potentials of bulk Ge. ${ }^{18}$ In the $s p^{3} d^{5} s^{*}$ TB method, one $s$ orbital, three $p$ orbitals $\left\{p_{x}, p_{y}, p_{z}\right\}$, five $d$ orbitals $\left\{d_{y z}, d_{z x}, d_{x y}, d_{3 z^{2}-r^{2}}, d_{x^{2}-y^{2}}\right\}$, and one excited $s^{*}$ orbital are considered. Spin-orbit coupling was taken into account in this study because it has large effect on the valence band structure of Ge. Surface dangling bonds were passivated by giving excess energy according to the $s p^{3}$ hybridization scheme. ${ }^{19}$

The phonon dispersion and the vibrational states were calculated by a valence force field (VFF) model ${ }^{20-22}$ with a free boundary condition. The adopted VFF parameters were those fitted to the phonon dispersion of bulk Ge. ${ }^{21}$ In this framework, the equation of motion of atoms constituting a NW is solved under atomistic description of potential energy, and all the phonon modes including both acoustic and optical modes are considered.

The electron-phonon interaction Hamiltonian was derived from the variation of TB Hamiltonian by the phonons, and the scattering rate was calculated by the Fermi's golden rule using the hole and phonon states computed as 
above. Then low-field hole mobility was calculated by solving linearized Boltzmann's transport equation, ${ }^{23}$ assuming a temperature of $300 \mathrm{~K}$ and using Fermi-Dirac distribution. Here, calculations for both low and high hole density were performed. At low hole density, the electrostatic potential in the NW is almost flat and it has negligible effect on the band structure. At the same time, the hole distribution function can be approximated by Boltzmann distribution, and the hole mobility does not depend on the Fermi level. Thus, for simplicity, we used band structures calculated with flat electrostatic potential without self-consistency, and Fermi level was set at the mid-gap of bulk Ge. For the hole mobility at high hole density, we computed the hole states both with and without self-consistent calculation of electrostatic potential, and compared the results. In this case, the Fermi-Dirac distribution is directly taken into account. The computation was performed by our custom-made program..$^{9,11,14}$

\section{B. Nanowire structures}

Targeted Ge NWs have diamond structure and rectangular cross-sections with a constant width of $2 \mathrm{~nm}$ and varied height of $2-10 \mathrm{~nm}$. Their transport orientations and two side faces are $[001] /(100) /(010),[110] /(001) /(1 \overline{1} 0),[111] /(\overline{1} \overline{1} 2) /$ $(1 \overline{1} 0)$, and $[112] /(\overline{1} 1) /(1 \overline{1} 0)$. These crystal structures are shown in Fig. 1. The lattice constant $\left(a_{0}\right)$ was assumed to be the same as that of bulk Ge.

\section{RESULTS AND DISCUSSION}

\section{A. Valence band structure}

First, we show the calculated valence band structures of [001], [110], [111], and [112]-oriented Ge NWs with $2 \mathrm{~nm} \times 2 \mathrm{~nm}$ and $2 \mathrm{~nm} \times 6 \mathrm{~nm}$ cross sections in Figs. 2-5. (a) [001] Nanowire

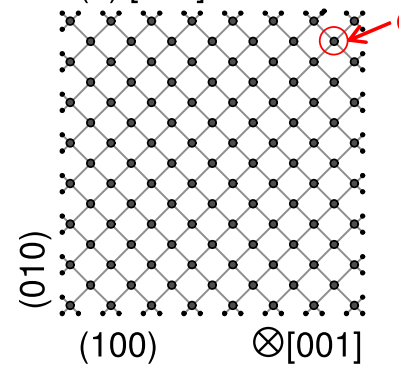

(c) [111] Nanowire

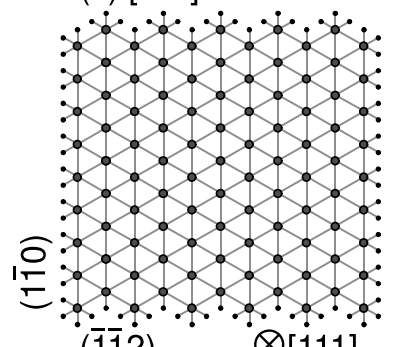

(112) $\otimes[111]$ (b) [110] Nanowire

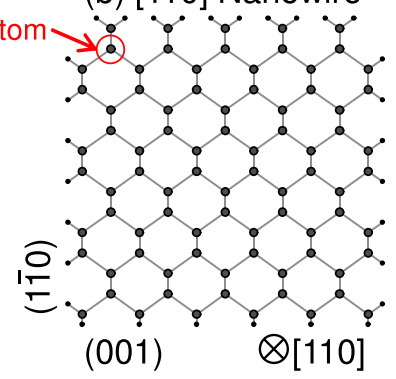

(d) [112] Nanowire

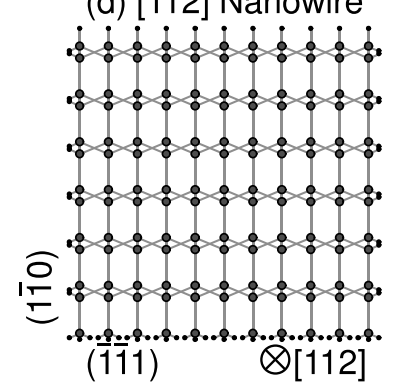

FIG. 1. Crystal structures of targeted Ge NWs. Each figure illustrates (a) $[001] /(100) /(010)$, (b) $[110] /(001) /(1 \overline{1} 0)$, (c) $[111] /(\overline{1} \overline{1} 2) /(1 \overline{1} 0)$, and (d) $[112] /(\overline{1} \overline{1} 1) /(1 \overline{1} 0)$ NWs. (a)

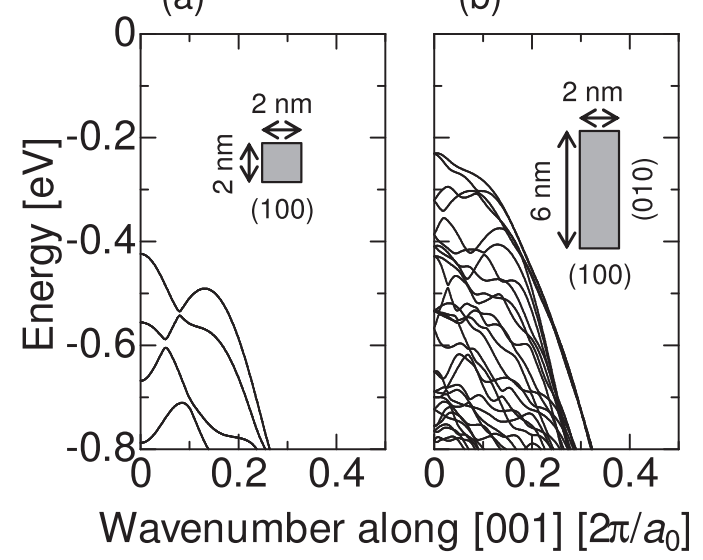

FIG. 2. The calculated valence band structures of [001] Ge NWs with (a) $2 \mathrm{~nm} \times 2 \mathrm{~nm}$ and (b) $2 \mathrm{~nm} \times 6 \mathrm{~nm}$ cross section.

The energy is referenced from the valence band maximum (VBM) of bulk Ge. All of the valence band structures have the VBM at the $\Gamma$ point, and the effective mass and confinement-induced energy shift depend on wire geometry. The VBM of the [110] Ge NWs with strong confinement along $[1 \overline{1} 0]$ direction ([110]/(001) NWs, Figs. 3(a) and 3(b)) and that of [111] NWs (Fig. 4) are characterized by light effective mass (0.06-0.07 $m_{0}$, where $m_{0}$ is the rest mass of an electron). On the other hand, the [110]/(110) NW (Fig. 3(c)) has heavier effective mass $\left(0.09 m_{0}\right)$ at VBM. The anisotropic effect of quantum confinement on holes can be understood from the viewpoint of contribution of atomic orbitals to the wave function. ${ }^{14}$ The effective mass of $[112] /(1 \overline{1} 0)$ NWs (Figs. 5(a) and $5(\mathrm{c}))$ is similar to that of the $[110] /(1 \overline{1} 0) \mathrm{NW}$, and [112]/(1̄11) (Fig. 5(b)) and [001] (Fig. 2) NWs have further heavier effective mass.

To analyze the geometry dependence of the valence band structure, we calculated the averaged hole effective mass $m_{\text {avg. }} m_{\text {avg }}$ is defined as the reciprocal number of inverse effective mass averaged by equilibrium hole distribution $\left[1-f^{0}\left(E_{n}(k)-E_{\mathrm{F}}\right)\right]$, as given by

$$
\frac{1}{m_{\mathrm{avg}}} \equiv \frac{\sum_{n} \int_{-k_{\mathrm{BZE}}}^{k_{\mathrm{BZE}}} \frac{1}{-m_{n}^{*}(k)}\left[1-f^{0}\left(E_{n}(k)-E_{\mathrm{F}}\right)\right] d k}{\sum_{n} \int_{-k_{\mathrm{BZE}}}^{k_{\mathrm{BZE}}}\left[1-f^{0}\left(E_{n}(k)-E_{\mathrm{F}}\right)\right] d k},
$$

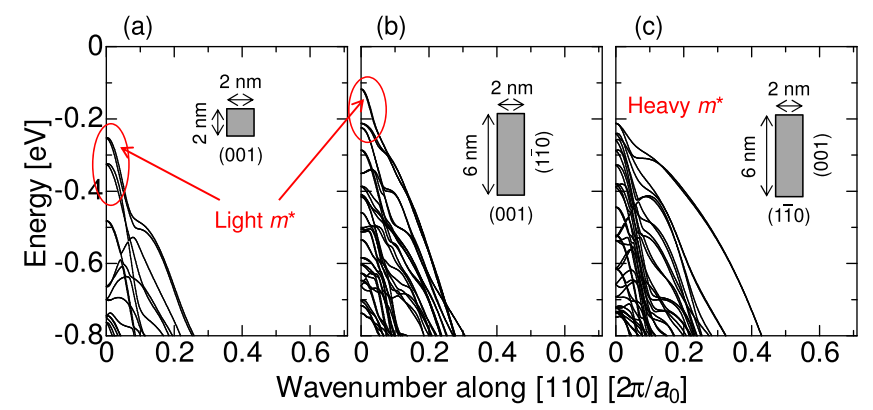

FIG. 3. The calculated valence band structures of [110] Ge NWs with (a) $2 \mathrm{~nm} \times 2 \mathrm{~nm}$ and (b) $2 \mathrm{~nm} \times 6 \mathrm{~nm}$ cross section on (001), and (c) $2 \mathrm{~nm} \times 6 \mathrm{~nm}$ cross section on (110). 
(a)

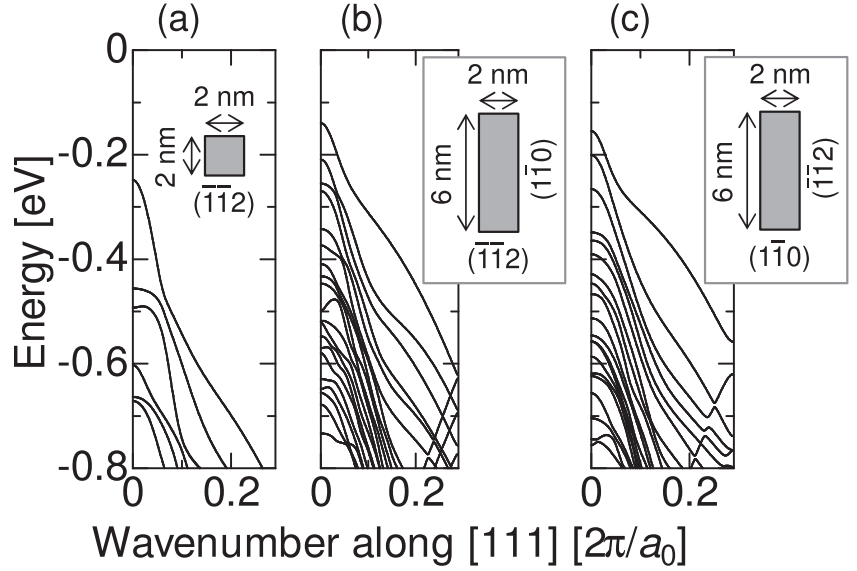

FIG. 4. The calculated valence band structures of [111] Ge NWs with (a) $2 \mathrm{~nm} \times 2 \mathrm{~nm}$ and (b) $2 \mathrm{~nm} \times 6 \mathrm{~nm}$ cross section on (112), and (c) $2 \mathrm{~nm} \times 6 \mathrm{~nm}$ cross section on $(1 \overline{1} 0)$.

where $n, k, k_{\mathrm{BZE}}$, and $m_{n}^{*}(k)$ are the subband index, the wavenumber of an electron, the wavenumber at the first Brillouin zone edge of NWs, and the effective mass at a given $k$ as an electron. Discussion on the physical meaning of $m_{\mathrm{avg}}$ is given in the Appendix. The distribution function is generally the Fermi-Dirac distribution. However, since we now consider the case with low hole density and Fermi level at the mid-gap, Boltzmann distribution can be used as the distribution function of holes.

Calculated $m_{\text {avg }}$ is shown in Fig. 6 for rectangular crosssectional Ge NWs with a constant width of $2 \mathrm{~nm}$ and varied height from 2 to $10 \mathrm{~nm}$. From Fig. 6, we can see that $m_{\text {avg }}$ tends to increase as the NW height increases except for [110]/(001) NWs. This increase of $m_{\mathrm{avg}}$ is due to reduced quantum confinement on heavy-transport-mass components of hole states. ${ }^{14}$

\section{B. Phonon band structure}

Next, some examples of calculated phonon band structures (dispersion relations) in Ge NWs are presented in Fig. 7. In contrast to the phonon band of bulk Ge, many subbands can be seen, corresponding to the increased degree of

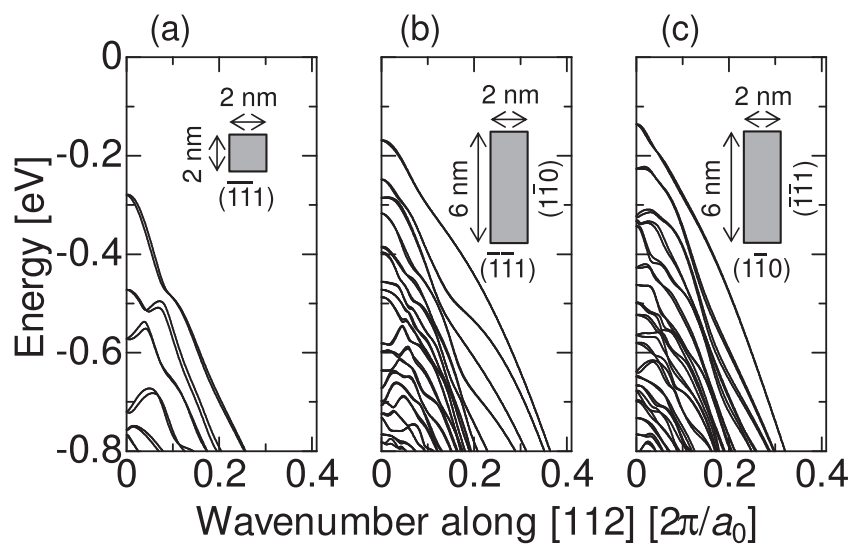

FIG. 5. The calculated valence band structures of [112] Ge NWs with (a) $2 \mathrm{~nm} \times 2 \mathrm{~nm}$ and (b) $2 \mathrm{~nm} \times 6 \mathrm{~nm}$ cross section on (111), and (c) $2 \mathrm{~nm} \times 6 \mathrm{~nm}$ cross section on $(1 \overline{1} 0)$.

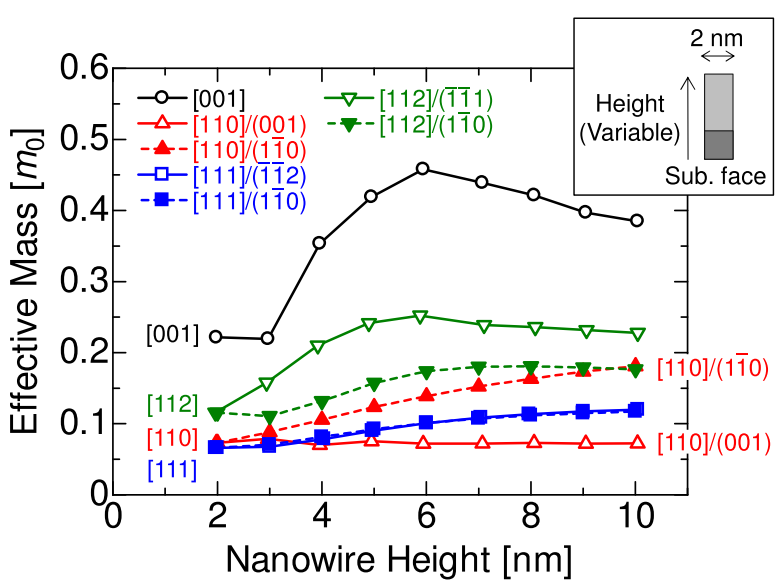

FIG. 6. Height dependence of average hole effective mass in rectangular cross-sectional Ge NWs with different orientations and substrate faces. The width along the substrate face was kept at $2 \mathrm{~nm}$ and the height vertical to the substrate face was varied.

freedom about the motion of atoms in the large unit cell of NWs. Subbands with energy below $30 \mathrm{meV}$ are mainly derived from acoustic phonons of bulk Ge, and subbands with higher energy originate from optical phonons.

To investigate the contribution of phonons within different energy ranges, we calculated mobilities at low hole density in $2 \mathrm{~nm} \times 2 \mathrm{~nm}$ and $2 \mathrm{~nm} \times 6 \mathrm{~nm}$ NWs with various orientations and substrate faces using phonons in intentionally limited energy ranges. When we used phonons with energy below $30 \mathrm{meV}$, the inverse of mobility $(1 / \mu$, as an index of strength of scattering) was about $70 \%-80 \%$ (depending on geometries) of that calculated in the same NW considering all phonons. This indicates that the acoustic phonons are dominant, although the optical phonons cannot be neglected, in particular, for holes with sufficient energy to (a) $[110]$

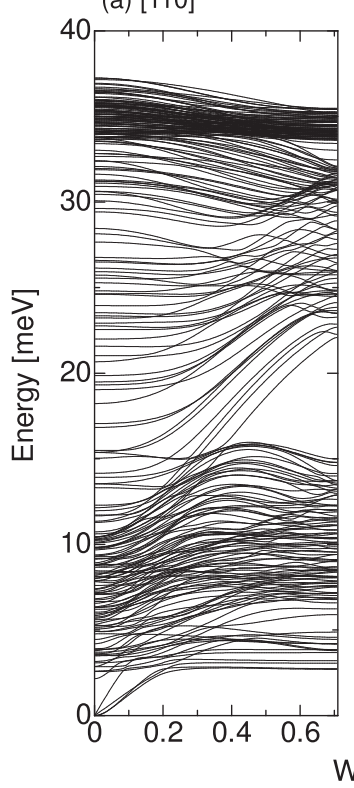

(b) $[110] /(001)$

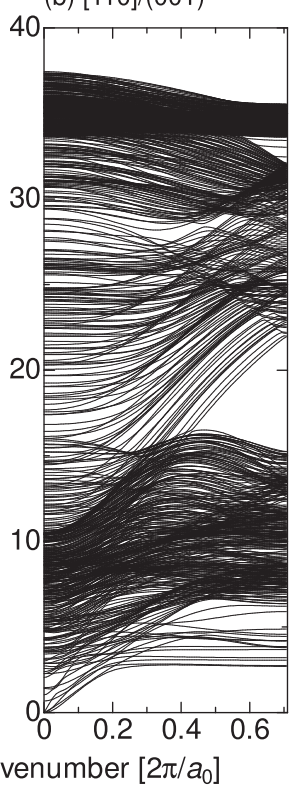

(c) [111]

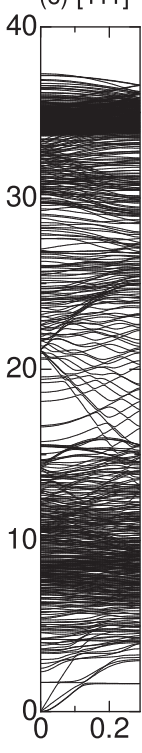

FIG. 7. The calculated dispersion relations of phonons in (a) [110] Ge NW with $2 \mathrm{~nm} \times 2 \mathrm{~nm}$ cross section, (b) [110] Ge NW with $2 \mathrm{~nm} \times 6 \mathrm{~nm}$ cross section on (001), and (c) [111] Ge NW with $2 \mathrm{~nm} \times 2 \mathrm{~nm}$ cross section. 
emit optical phonons. When the upper limit of phonon energy was lowered from $30 \mathrm{meV}$ to $20 \mathrm{meV}$, the $1 / \mu$ was almost the same, showing negligible effect of $20-30 \mathrm{meV}$ phonons.

In view of the geometrical effects on hole transport properties, it should be noted that the dependence of the phonon band structures on wire geometry (except for the increase of subbands with the increase of the volume of the unit cell) is small compared to those of valence band structures. In particular, the dependence of density of states per unit volume on wire geometry is weak. Therefore, the dependence of phonon band structure on orientations and substrate faces should have small effects on hole transport properties.

\section{Hole mobility at low hole density}

Figure 8(a) depicts the height dependence of the computed phonon-limited hole mobility $\mu$ in various Ge NWs with rectangular cross sections. The Fermi level is fixed at the mid-gap also in this calculation. Among square crosssectional NWs with $2 \mathrm{~nm}$ height, the [111] NW showed the highest $\mu$. This is consistent with a previous report on hole mobility in circular cross-sectional Ge NWs. ${ }^{10}$ As the NW height increases, $\mu$ of $[110] /(001)$ NWs significantly increases. When the height is larger than $4 \mathrm{~nm}$, it exceeds the bulk mobility calculated by a similar approach $\left(2280 \mathrm{~cm}^{2} / \mathrm{V} \cdot \mathrm{s}\right) .{ }^{10}$ On the other hand, NWs with other geometries have small height dependence of mobility. By comparing $\mu$ (Fig. 8(a)) with $m_{\text {avg }}$ (Fig. 6), we can confirm the expectable tendency that NWs with high $\mu$ have light $m_{\text {avg. }}$. However, the geometry dependence of $\mu$ cannot be explained only by the information of $m_{\text {avg. }}$.

Then, to further clarify the factor determining the geometry dependence of $\mu$, we defined an average relaxation time $\langle\tau\rangle$ from the following equation:

$$
\mu=\frac{e\langle\tau\rangle}{m_{\mathrm{avg}}},
$$

where $e$ is the elementary charge ( $>0$ ). The calculated average relaxation time $\langle\tau\rangle$ is shown in Fig. 8(b). Since $\langle\tau\rangle$ does not show direct correlation with $m_{\text {avg }}$, we analyzed $\langle\tau\rangle$ using other information of the valence band structure than $m_{\mathrm{avg}}$.
First, we can expect $\langle\tau\rangle$ is linked to the density of states (DOS) of holes, because large DOS means a large number of possible final states for scattering. Thus, we defined $N_{\text {avg }}$ (DOS integrated with a Boltzmann factor) as

$$
N_{\text {avg }} \equiv \int_{-\infty}^{E_{\mathrm{v}}} \operatorname{DOS}(E) \exp \left(-\frac{E_{\mathrm{v}}-E}{k_{\mathrm{B}} T}\right) d E,
$$

where $\operatorname{DOS}(E), E_{\mathrm{v}}, k_{\mathrm{B}}$, and $T$ are the DOS per unit length, the energy of VBM, the Boltzmann constant, and the temperature $(300 \mathrm{~K})$, respectively.

At the same time, electron-phonon interaction is enhanced approximately in proportion to $1 / S$, where $S$ is the cross-sectional area of NWs. ${ }^{24}$ This phenomenon can be understood in the confined phonon picture as follows. In thinner NWs, the number of atoms in their unit cell decreases, thus the displacement of each atom increases for a mode with the same phonon energy, because fewer atoms keep the same total vibration energy. Though at the same time the number of phonon modes (subbands) decreases in thinner NWs, this effect is canceled by the relaxation of the wavenumber conservation rule inside the cross-sectional plane.

Following the above discussion, we calculated the geometry dependence of $S / N_{\text {avg }}$ and compared it with $\langle\tau\rangle$ (Fig. 9). Although the details of phonons and electronphonon coupling are ignored in $S / N_{\text {avg }}$, the geometry dependence of $\langle\tau\rangle$ is basically reproduced by the geometry dependence of $S / N_{\mathrm{avg}}$, and strong correlation between $\langle\tau\rangle$ and $S / N_{\mathrm{avg}}$ can be confirmed (Fig. 9(b)). Therefore, $\langle\tau\rangle$ can be mainly determined by the DOS of holes and the cross-sectional area of NWs. This result means that the geometry dependence of $\mu$ calculated using atomistic hole and phonon states can be qualitatively explained simply by the information of the valence band structure (effective mass $\rightarrow m_{\mathrm{avg}}$ and DOS $\rightarrow\langle\tau\rangle)$ and the cross-sectional area of NWs.

In the following, the behavior of $\mu$ in various geometries is discussed. In [110]/(001) NWs, when $S$ (or NW height) increases, $m_{\text {avg }}$ stays light. At the same time, $N_{\text {avg }}$ keeps small (height $<8 \mathrm{~nm}$ ) and thus $S / N_{\text {avg }}$ increases, which means the increase of $\langle\tau\rangle$ and $\mu=e\langle\tau\rangle / m_{\text {avg. }}$. When the height is further increased, the subband separation becomes small and thus $N_{\text {avg }}$ increases. This cancels the $S$ increase and therefore $\langle\tau\rangle$ and $\mu$ are expected to converge. In fact,

(b)

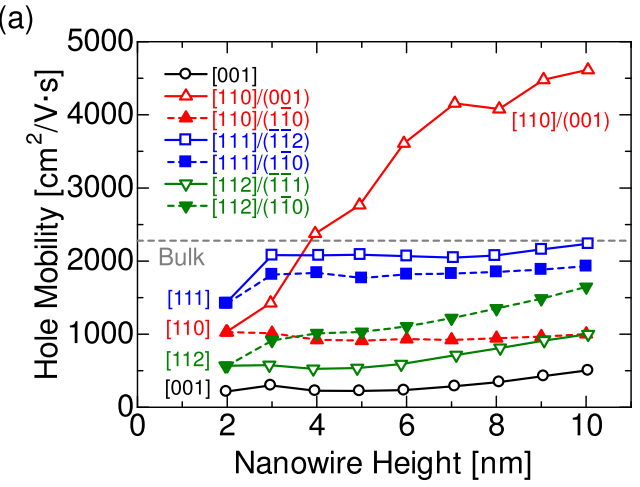

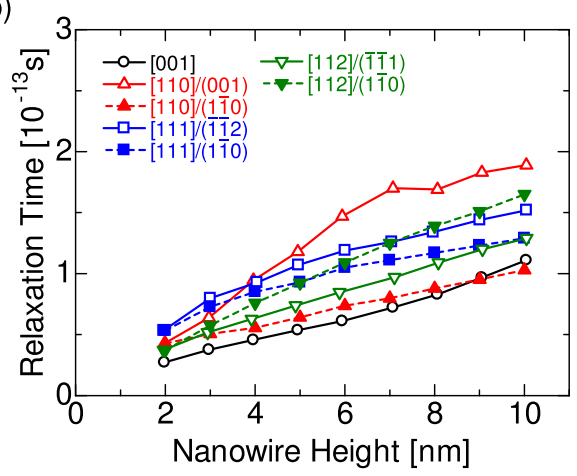

FIG. 8. (a) Height dependence of the calculated phonon-limited hole mobility in various Ge NWs. The width was kept at $2 \mathrm{~nm}$ (common to (a) and (b)). The gray horizontal broken line shows the bulk mobility calculated in a similar method. ${ }^{10}$ (b) Height dependence of the average relaxation time in various Ge NWs derived from the relation between the average effective mass and the mobility (Eq. (2)). 
(a)

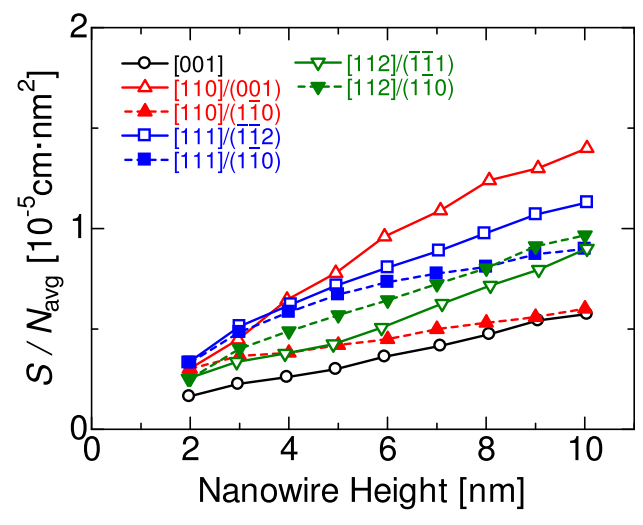

(b)

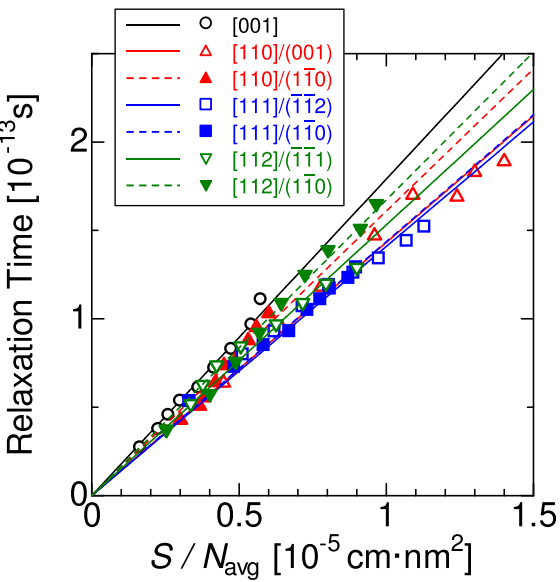

FIG. 9. (a) Height dependence of cross-sectional area $(S)$ divided by density of states integrated with a Boltzmann factor $\left(N_{\mathrm{avg}}\right)$ in various $\mathrm{Ge}$ NWs. (b) Comparison between the average relaxation time $\langle\tau\rangle$ and $S / N_{\text {avg. }}$. Lines are least-square fits. Strong correlation between $\langle\tau\rangle$ and $S / N_{\text {avg }}$ can be confirmed.
[110]/(001) NWs with 20 and $30 \mathrm{~nm}$ height exhibited $\mu$ of 5159 and $5315 \mathrm{~cm}^{2} / \mathrm{V} \cdot \mathrm{s}$, respectively. On the contrary, $N_{\mathrm{avg}}$ and $m_{\text {avg }}$ of [111] NWs become larger as the height (and $S$ ) increases. Thus, the increase of $S$ is canceled and $\mu$ (approximately proportional to $\left.S /\left(N_{\mathrm{avg}} m_{\mathrm{avg}}\right)\right)$ stays almost constant. $[110] /(1 \overline{1} 0)$ NWs show shorter $\langle\tau\rangle$ and lower $\mu$ than $[112] /(1 \overline{1} 0)$ NWs due to their larger DOS $\left(N_{\mathrm{avg}}\right)$, in spite of

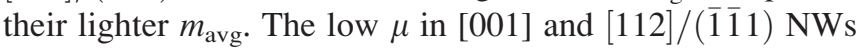
is ascribed to their heavy $m_{\mathrm{avg}}$ and large DOS $\left(N_{\mathrm{avg}}\right)$.

\section{Hole density dependence of hole mobility}

Though we have discussed the hole mobility at low hole density above, the mobility at high hole density is also important because the carrier density becomes high in the on-state of FETs. Thus, we calculated the hole density dependence of hole mobility $\mu$ in Ge NWs. The cross-sectional shape of NWs is fixed at a rectangle with 2-nm width and 6$\mathrm{nm}$ height, and NWs with various orientations and substrate faces were considered. The calculated results are shown in Fig. 10. In this figure, lines present the mobility calculated assuming a flat electrostatic potential in NWs (without selfconsistency (SC)), in the same way as the above case at low hole density. In this case, only the Fermi level was varied without changing the valence band structure. In contrast,

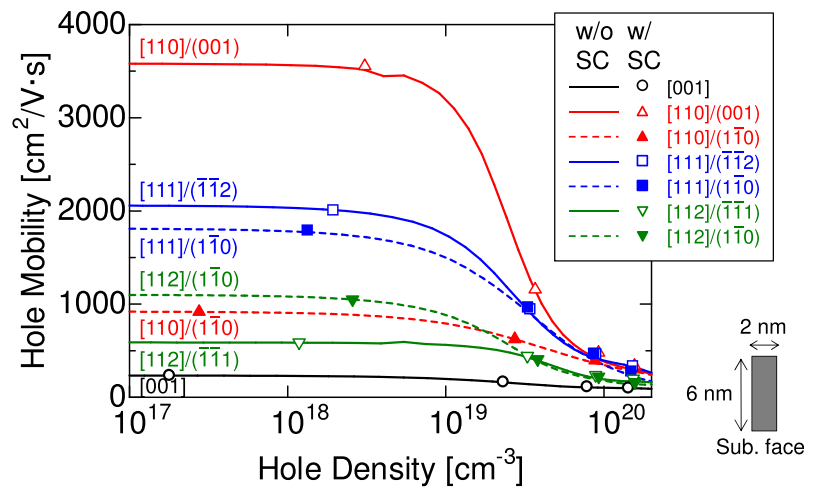

FIG. 10. Hole density dependence of mobility in rectangular cross-sectional Ge NWs with different orientations and substrate faces. Considered NWs have the width of $2 \mathrm{~nm}$ and the height of $6 \mathrm{~nm}$. Results with and without SC are presented by symbols and lines, respectively. data shown by symbols were calculated using the valence band structure computed self-consistently with the Poisson equation, which gives the electrostatic potential taking account of the charge density (with SC). We assumed $0.6 \mathrm{~nm}$-thick $\mathrm{SiO}_{2}$ as a gate oxide for calculation with SC. The Fermi level in this case was fixed at $0 \mathrm{eV}$ (the VBM of bulk $\mathrm{Ge}$ at the electrostatic potential of $0 \mathrm{~V}$ ), and the gate voltage was defined as the shift of the electrostatic potential at the perimeter of gate oxide from the initial flat potential of $0 \mathrm{~V}$. The symbols in Fig. 10 correspond to the gate voltage of $-0.1,-0.3,-0.5$, and $-0.7 \mathrm{~V}$.

From Fig. 10, we can see that the $[110] /(001)$ NW and [111] NWs, which showed high $\mu$ at low hole density, also exhibit higher $\mu$ than other NWs even at high hole density. However, the $\mu$ degrades as the hole density increases, especially in the $[110] /(001) \mathrm{NW}$. This trend is common to $\mu$ with and without SC, and Fig. 10 also shows that the difference between results with and without SC is small in such thin NWs with a cross-sectional size of $2 \mathrm{~nm} \times 6 \mathrm{~nm}$. Thus, the hole density dependence of $\mu$ can be attributed to the change of hole occupancy as the hole density increases, not to a change of the valence band structure as the gate voltage increases.

To analyze the dependence of $\mu$ on hole density and geometry, we again computed $m_{\mathrm{avg}}$ and $\langle\tau\rangle=m_{\mathrm{avg}} \mu / e$ (Fig. 11). The definition of $m_{\text {avg }}$ (Eq. (1)) is validated even in degenerate hole distribution as follows: If all the hole states have a constant relaxation time of $\tau_{c}$, the mobility $\mu$ can be written as $e \tau_{c} / m_{\text {avg }}$ regardless of hole density (the Appendix). Thus, $m_{\text {avg }}$ behaves as the "effective" hole mass. As shown in Fig. 11(a), $m_{\text {avg }}$ increases as the hole density increases. This result can be ascribed to the contribution of heavy-effective-mass subbands separated from VBM by quantum confinement. At the same time, $\langle\tau\rangle$ decreases as the hole density increases (Fig. 11(b)). This can be explained by the increase of the number of subbands contributing to conduction, which causes the increase of the DOS near the Fermi level, in other words, the DOS in the energy region dominating the carrier transport.

Fig. 11(b) also shows that the geometry dependence of $\langle\tau\rangle$ is small at high hole density $\left(>1 \times 10^{20} \mathrm{~cm}^{-3}\right)$. Although it must be noted that the small geometry dependence of $\langle\tau\rangle$ is seen for NWs with the same cross-sectional 
(a)

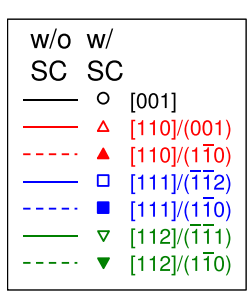

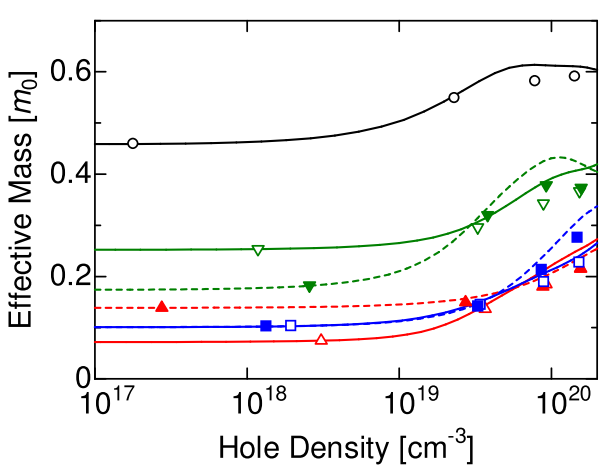

(b)

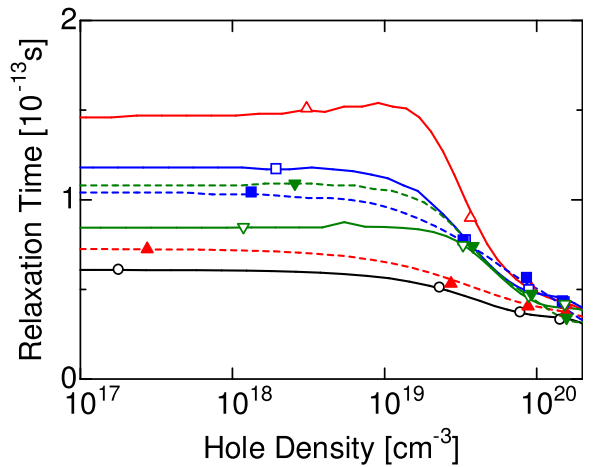

FIG. 11. Hole density dependence of (a) average hole effective mass $m_{\text {avg }}$ and (b) average relaxation time $\langle\tau\rangle$ in various Ge NWs. At high hole density, $m_{\text {avg }}$ becomes large but geometry dependence is maintained. In contrast, $\langle\tau\rangle$ decreases at high density and becomes almost independent of the wire geometry.

area, this implies that the dependence of $\mu$ on the orientations and substrate faces at high hole density is mainly determined by the difference in the effective mass, not by the relaxation time.

Although this paper discussed the phonon-limited hole mobility, carrier transport may be affected significantly by the surface roughness scattering in strong inversion regime or under strong quantum confinement. This will be investigated in our future study.

\section{CONCLUSION}

We calculated the phonon-limited hole mobility in Ge NWs with rectangular cross-sections and various orientations based on atomistic approaches, a tight-binding approximation and a valence force field model. The analysis of the geometry dependence of hole mobility revealed that the dependence was explainable by an average effective mass and the density of states of holes. In the case of square crosssections, the [111] Ge NW showed the highest mobility, and for rectangular cross-sections, [110] Ge NWs with larger height along the [001] direction $([110] /(001)$ NWs) exhibited high hole mobility. Since [110]/(001) Ge NWs are also expected to have high electron mobility, ${ }^{11}$ they are promising for CMOS applications.

\section{APPENDIX: VALIDATION OF AVERAGE EFFECTIVE MASS}

In this section, we present the validity of the definition of average effective mass ( $m_{\text {avg }}$, Eq. (1)) and explain its physical meaning. Under relaxation time approximation, the hole mobility $\mu$ can be written as ${ }^{25}$

$\mu=\frac{e \sum_{n} \int_{-k_{\mathrm{BZE}}}^{k_{\mathrm{BZE}}}\left[\tau_{n}(k) \frac{d\left[1-f^{0}\left(E_{n}(k)-E_{\mathrm{F}}\right)\right]}{d k} \frac{1}{\hbar}\right] v_{n}(k) \frac{d k}{2 \pi}}{\sum_{n} \int_{-k_{\mathrm{BZE}}}^{k_{\mathrm{BZE}}}\left[1-f^{0}\left(E_{n}(k)-E_{\mathrm{F}}\right)\right] \frac{d k}{2 \pi}}$

$=\frac{I /(e F)}{n_{\mathrm{h}}}$, where $\tau_{n}(k), v_{n}(k)$, and $f^{0}\left(E_{n}(k)-E_{\mathrm{F}}\right)$ are the momentum relaxation time, group velocity $\left(=\hbar^{-1} d E_{n} / d k\right)$, and equilibrium distribution function (Fermi-Dirac distribution) of electrons, respectively. The spin degree of freedom is treated as different subbands, and thus the density of states in $k$-space is $1 / 2 \pi$. In the right hand side of Eq. (A1), $F$ is a spatially uniform and small electric field applied to the system, and $n_{\mathrm{h}}$ is hole density per unit length. Current $I$ can be expressed as $e\left\langle v_{\mathrm{h}}\right\rangle n_{\mathrm{h}}$, using average hole drift velocity $\left\langle v_{\mathrm{h}}\right\rangle$.

Then, the numerator $I /(e F)$ is rewritten to an expression including effective mass by partial integration

$$
\begin{aligned}
I /(e F)= & e \sum_{n} \int_{-k_{\mathrm{BZE}}}^{k_{\mathrm{BZE}}}\left[\tau_{n}(k) \frac{d\left[1-f^{0}\left(E_{n}(k)-E_{\mathrm{F}}\right)\right]}{d k} \frac{1}{\hbar^{2}} \frac{d E_{n}}{d k}\right] \\
& \times \frac{d k}{2 \pi}, \\
= & e \sum_{n} \frac{1}{2 \pi}\left[\tau_{n}(k)\left[1-f^{0}\left(E_{n}(k)-E_{\mathrm{F}}\right)\right] \frac{1}{\hbar^{2}} \frac{d E_{n}}{d k}\right]_{-k_{\mathrm{BZE}}}^{k_{\mathrm{BZE}}} \\
& -e \sum_{n} \int_{-k_{\mathrm{BZE}}}^{k_{\mathrm{BZE}}}\left[\left[1-f^{0}\left(E_{n}(k)-E_{\mathrm{F}}\right)\right]\right. \\
& \left.\times \frac{d}{d k}\left(\tau_{n}(k) \frac{1}{\hbar^{2}} \frac{d E_{n}}{d k}\right)\right] \frac{d k}{2 \pi} .
\end{aligned}
$$

The first term becomes zero because of the periodicity of the first Brillouin zone. Furthermore, assuming the relaxation time $\tau_{n}(k)$ as independent of $k$ and $n$, and defining $\tau_{n}(k)$ $=\tau_{c}$, we have

$$
\begin{aligned}
I /(e F)= & 0-e \sum_{n} \int_{-k_{\mathrm{BZE}}}^{k_{\mathrm{BZE}}}\left[\left[1-f^{0}\left(E_{n}(k)-E_{\mathrm{F}}\right)\right]\right. \\
& \left.\times \frac{d}{d k}\left(\tau_{c} \frac{1}{\hbar^{2}} \frac{d E_{n}}{d k}\right)\right] \frac{d k}{2 \pi}, \\
= & e \sum_{n} \int_{-k_{\mathrm{BZE}}}^{k_{\mathrm{BZE}}}\left[\left[1-f^{0}\left(E_{n}(k)-E_{\mathrm{F}}\right)\right] \tau_{c} \frac{1}{-m_{n}^{*}(k)}\right] \frac{d k}{2 \pi},
\end{aligned}
$$

where $1 / m_{n}^{*}(k)=\hbar^{-2} d^{2} E_{n} / d k^{2}$. Substituting this into Eq. (A1), $\mu$ is expressed as 


$$
\begin{aligned}
\mu & =\frac{e \tau_{c} \sum_{n} \int_{-k_{\mathrm{BZE}}}^{k_{\mathrm{BZE}}}\left[\left[1-f^{0}\left(E_{n}(k)-E_{\mathrm{F}}\right)\right] \frac{1}{-m_{n}^{*}(k)}\right] d k}{\sum_{n} \int_{-k_{\mathrm{BZE}}}^{k_{\mathrm{BZE}}}\left[1-f^{0}\left(E_{n}(k)-E_{\mathrm{F}}\right)\right] d k} \\
= & \frac{e \tau_{c}}{m_{\mathrm{avg}}} .
\end{aligned}
$$

This indicates that $m_{\text {avg }}$ defined in Eq. (1) behaves as the effective hole mass under the assumption of constant relaxation time. Also, it is found that the average relaxation time $\langle\tau\rangle$ defined in Eq. (2) corresponds to this constant relaxation time $\tau_{c}$ which can reproduce the computed value of $\mu$.

It is of interest that this result is derived without assuming non-degenerate hole distribution. In the degenerate case, strongly non-parabolic subbands above $E_{\mathrm{F}}$ (occupied by holes) may have both states with positive and negative effective masses, but they are canceled in the calculation of average effective mass. The average effective mass has only the information of group velocity near $E_{\mathrm{F}}$ (Eq. (A1)), ${ }^{25}$ which dominates the carrier transport. Because completely occupied states do not contribute to conduction, a constant average relaxation time $\langle\tau\rangle\left(=\tau_{c}\right)$ can be determined around $E_{\mathrm{F}}$.

${ }^{1}$ See http://www.itrs.net for International Technology Roadmap for Semiconductor, 2013.

${ }^{2}$ K. Saraswat, C. Chui, T. Krishnamohan, A. Nayfeh, and P. McIntyre, Microelectron. Eng. 80, 15 (2005).

${ }^{3}$ R. Zhang, P. Huang, J. Lin, N. Taoka, M. Takenaka, and S. Takagi, IEEE Trans. Electron Devices 60, 927 (2013).
${ }^{4}$ C. H. Lee, C. Lu, T. Nishimura, K. Nagashio, and A. Toriumi, Symp. VLSI Technol. Dig. Tech. Papers 2014, 144-145.

${ }^{5}$ J.-T. Park and J.-P. Colinge, IEEE Trans. Electron Devices 49, 2222 (2002).

${ }^{6}$ N. Singh, A. Agarwal, L. Bera, T. Liow, R. Yang, S. Rustagi, C. Tung, R. Kumar, G. Lo, N. Balasubramanian, and D. Kwong, IEEE Electron Device Lett. 27, 383 (2006).

${ }^{7}$ J. Wang, A. Rahman, G. Klimeck, and M. Lundstrom, Tech. Dig.- IEEE Int. Electron Device Meet. 2005, 530-533.

${ }^{8}$ H. Minari and N. Mori, Jpn. J. Appl. Phys., Part 1 49, 04DN04 (2010).

${ }^{9}$ S. Mori, N. Morioka, J. Suda, and T. Kimoto, IEEE Trans. Electron Devices 60, 944 (2013).

${ }^{10}$ Y.-M. Niquet and C. Delerue, J. Appl. Phys. 112, 084301 (2012).

${ }^{11}$ H. Tanaka, S. Mori, N. Morioka, J. Suda, and T. Kimoto, IEEE Trans. Electron Devices 61, 1993 (2014).

${ }^{12}$ J.-A. Yan, L. Yang, and M. Y. Chou, Phys. Rev. B 76, 115319 (2007).

${ }^{13}$ N. Neophytou, A. Paul, and G. Klimeck, IEEE Trans. Nanotechnol. 7, 710 (2008).

${ }^{14}$ N. Morioka, H. Yoshioka, J. Suda, and T. Kimoto, J. Appl. Phys. 109, 064318 (2011).

${ }^{15}$ S. Koba, R. Ishida, Y. Kubota, H. Tsuchiya, Y. Kamakura, N. Mori, and M. Ogawa, Tech. Dig.- IEEE Int. Electron Device Meet. 2013, 312-315.

${ }^{16}$ J. C. Slater and G. F. Koster, Phys. Rev. 94, 1498 (1954).

${ }^{17}$ J. M. Jancu, R. Scholz, F. Beltram, and F. Bassani, Phys. Rev. B 57, 6493 (1998).

${ }^{18}$ Y.-M. Niquet, D. Rideau, C. Tavernier, and H. Jaouen, Phys. Rev. B 79, 245201 (2009).

${ }^{19}$ S. Lee, F. Oyafuso, P. Allmen, and G. Klimeck, Phys. Rev. B 69, 045316 (2004).

${ }^{20}$ P. N. Keating, Phys. Rev. 145, 637 (1966).

${ }^{21}$ Z. Sui and I. P. Herman, Phys. Rev. B 48, 17938 (1993).

${ }^{22}$ A. Paul, M. Luisier, and G. Klimeck, J. Comput. Electron. 9, 160 (2010).

${ }^{23}$ W. Zhang, C. Delerue, Y.-M. Niquet, G. Allan, and E. Wang, Phys. Rev. B 82, 115319 (2010).

${ }^{24}$ J. Lee and M. O. Vassell, J. Phys. C: Solid State Phys. 17, 2525 (1984).

${ }^{25}$ R. Kotlyar, T. D. Linton, R. Rios, M. D. Giles, S. M. Cea, K. J. Kuhn, M. Povolotskyi, T. Kubis, and G. Klimeck, J. Appl. Phys. 111, 123718 (2012). 